\title{
Fast 2-D and 3-D Terahertz Imaging With a Quantum-Cascade Laser and a Scanning Mirror
}

\author{
Nick Rothbart, Heiko Richter, Martin Wienold, Lutz Schrottke, Holger T. Grahn, and Heinz-Wilhelm Hübers
}

\begin{abstract}
A terahertz imaging system based on a quantum-cascade laser (QCL), a fast scanning mirror, and a sensitive Ge:Ga detector is demonstrated. Transmission images are obtained by scanning the beam of the QCL across an object. Images with a diameter of approximately $40 \mathrm{~mm}$ and a signal-to-noise ratio of up to 28 $\mathrm{dB}$ were obtained within $1.1 \mathrm{~s}$. The system was also used to obtain three-dimensional images of objects in an ellipsoidal volume with axes of approximately $40 \mathrm{~mm}$ by computed tomography within 87 s.
\end{abstract}

Index Terms-Computed tomography, imaging, quantum-cascade laser (QCL), scanning mirror, terahertz $(\mathrm{THz})$.

\section{INTRODUCTION}

I MAGING with terahertz $(\mathrm{THz})$ waves is very promising for many applications, e.g. in biomedicine, non-destructive testing or security. In biomedicine, the high absorption of $\mathrm{THz}$ radiation by water can be exploited, since for example the presence of cancer causes a locally higher water content of the biological tissue [1]. Furthermore, in vivo detection of breast cancer was demonstrated with a mouse model [2]. In non-destructive testing, the capabilities of THz imaging were proven by the detection of defects in the thermal insulation of the space shuttle [3]. For security applications, stand-off $\mathrm{THz}$ imaging is important because $\mathrm{THz}$ radiation penetrates many clothing materials and allows for the detection of hidden objects. Such systems are either active, i.e. with illumination by a $\mathrm{THz}$ radiation source [4], or passive, i.e. the imaging system detects only the natural $\mathrm{THz}$ radiation [5]. Because many explosives have characteristic spectra in the $\mathrm{THz}$ frequency range, spectroscopic imaging, i.e. obtaining spectral information along with the image, is of particular importance [6], [7].

A number of $\mathrm{THz}$ imaging approaches have already been demonstrated. This includes camera-like stand-off imaging,

Manuscript received January 25, 2013; revised March 27, 2013; accepted July 08, 2013. Date of publication August 06, 2013; date of current version September 18, 2013. This work was supported in part by the Investitionsbank Berlinunder Grants 10146488 and 10146490 within the EFRE program of the European Union. The work of N. Rothbart was supported by the Helmholtz Research School on Security Technologies.

N. Rothbart and H. Richter are with the Institute of Panetary Research, German Aerospace Center (DLR), 12489 Berlin, Germany (e-mail: nick.rothbart@dlr.de).

M. Wienold, L. Schrottke, and H. T. Grahn are with Paul-Drude-Institut für Festkörperelektronik, 10117 Berlin, Germany.

H.-W. Hübers is with the Institute of Planetary Research, German Aerospace Center (DLR), 12489 Berlin, Germany and Institut für Optik und Atomare Physik, Technische Universität Berlin, 10623 Berlin, Germany.

Color versions of one or more of the figures in this paper are available online at $\mathrm{http}: / /$ ieeexplore.ieee.org.

Digital Object Identifier 10.1109/TTHZ.2013.2273226 near-field microscopy, and short-range transmission or reflection imaging [8 and references therein]. In particular in short-range imaging, these approaches are often based on $\mathrm{THz}$ time-domain spectroscopy (TDS) systems, which can provide spectral information along with the image. In addition, depth information can be obtained by analyzing the time of flight of a THz pulse or by computed tomography (CT) [9], [10]. With a few exceptions, e.g. [11], the object to be imaged has to be raster scanned across the focus of the $\mathrm{THz}$ beam. Thus, the image acquisition times of TDS imaging systems are long, and the systems are not very practical for many applications.

Another approach is based on continuous-wave (CW) $\mathrm{THz}$ sources. This requires a powerful $\mathrm{THz}$ source, for example a backward wave oscillator [12]. Quantum-cascade lasers (QCLs) are also well suited for this task, because they are compact, easy-to-handle, and provide high output powers in CW mode. A number of $\mathrm{THz}$ systems for transmission as well as reflection imaging employing a QCL and different single pixel detectors have been demonstrated. Examples are systems with a liquidhelium-cooled bolometer [13], a Golay cell [14], a pyroelectric detector [15], a Schottky diode [16], or a Si-bolometer [17]. Recently, self-mixing in the QCL was used for $\mathrm{THz}$ reflection imaging [18]. Besides two-dimensional (2-D) imaging, threedimensional (3-D) imaging by CT has also been demonstrated with a QCL [14]. The single-pixel systems have in common that the object which is going to be imaged has to be raster scanned through the focus of the beam emitted by the QCL. This is a rather time-consuming procedure, and typical imaging times are up to several hours.

Real-time imaging with a QCL was realized using a commercial focal-plane array microbolometer camera for detection [19]-[22]. However, these systems seem to suffer from fringe patterns, which appear in the $\mathrm{THz}$ image. These are caused by diffraction, etalon effects, and interference [19], [22].

We report on a novel $\mathrm{THz}$ transmission imaging system, which is based on a fast scanning mirror, a QCL as the radiation source, and a sensitive single-pixel Ge:Ga photoconductive detector. The beam which is emitted by the QCL is raster scanned across the object by using the scanning mirror, and the transmitted radiation is focused onto the Ge:Ga detector. Since scanning mirrors allow for very fast scanning times and the Ge:Ga detector provides a correspondingly short response time, such a system allows for images with pixel numbers on the order of 10000 within approximately $1 \mathrm{~s}$. Such an imaging approach has the advantage that fringe artifacts do not appear in the images. The paper is organized as follows: first the imaging system and its components will be described. Then the optical performance will be discussed. In the following section, 2-D transmission images and 3-D CT measurements are presented. 


\section{EXPERIMENTAL SETUP}

A scheme of the imaging system is shown in Fig. 1. The THz radiation is generated by a QCL with a Fabry-Pérot resonator and a surface-plasmon waveguide. This waveguide design was chosen, because QCLs with surface-plasmon waveguides provide a very good beam profile, which can be transformed into a Gaussian-shaped beam [23], [24]. The active medium is based on a bound-to-continuum design similar to the one published in [25]. The QCL is mounted to the cold finger of a compact Stirling cooler (model: Ricor K535) [24]. The output power of the QCL is about $300 \mu \mathrm{W}$ at a temperature of $31 \mathrm{~K}$. The output window of the vacuum housing around the cold finger is made from high density polyethylene (HDPE). It is mounted at the Brewster angle in order to minimize reflections and standing waves. The bias current for the QCL was supplied by a homemade, battery-driven current supply, which is used for setting the DC driving current for the QCL and superimposing a small sinusoidal AC current with a frequency of $100 \mathrm{kHz}$. In total, three modes around $2.5 \mathrm{THz}$ separated by $26 \mathrm{GHz}$ are emitted, with the central mode about 5 and 10 times more intense than the other two modes. The beam of the QCL is collimated by means of a 50-mm diameter poly-4-methylpentene-1 (TPX) lens with a focal length of $90 \mathrm{~mm}$. The beam impinges on a flat scanning mirror (AXSYS Technologies Imaging Systems FSM) with a diameter of $35 \mathrm{~mm}$, which is located between the TPX lens and the object plane at an angle of $25^{\circ}$. The scanning mirror deflects the beam toward the object. At the same time, it can be tilted up to $\pm 3^{\circ}$ in the vertical as well as horizontal direction with a resolution of $2 \mu \mathrm{rad}$, which is approximately $0.004 \%$ of the maximum deflection. This corresponds to a spatial separation of about 1 $\mu \mathrm{m}$ in the object plane. The deflection of the steering mirror is driven by an electronic controller and read out by a data acquisition (DAQ) pad, which in turn is controlled by a LabVIEW program. The readout of the actual mirror position is necessary, because the scan trajectory is not reproducible to the precision required for a good spatial resolution when the mirror is moved very quickly. The HDPE lens with a focal length of $250 \mathrm{~mm}$ and a diameter of $76 \mathrm{~mm}$ focuses the beam in the object plane. The distance between the scanning mirror and the HDPE lens equals its focal length. In this configuration, the paths of the beams are parallel with respect to each other after passing through the lens. By steering the mirror, the beam waist is scanned across the object. A variety of scan trajectories are possible. In this study, we used either spiral scanning with a fixed object or line scanning with the object moving in the vertical direction with respect to the scan line. In addition, the object can be mounted on a rotation stage. This allows for images of the object at different angles, which is a prerequisite for 3-D imaging based on CT. The transmitted radiation is collected by an off-axis parabolic mirror with an asymmetric shape with dimensions of $160 \mathrm{~mm}$ and $290 \mathrm{~mm}$ in the horizontal ( $x$-axis, cf. Fig. 1) and vertical direction ( $y$-axis, cf. Fig. 1), respectively, and a focal length of $76 \mathrm{~mm}$. This mirror, which is placed $180 \mathrm{~mm}$ behind the object plane, focuses the radiation from a solid angle of $44^{\circ}$ onto a Ge:Ga photoconductive detector. The detector signal is fed into a phase-sensitive lock-in amplifier (model HF2 by Zurich Instruments), which uses the $100 \mathrm{kHz}$ current modulation of the QCL as a reference, and read out by the DAQ pad. The sample rate of each writing and reading channel of the DAQ pad is 500

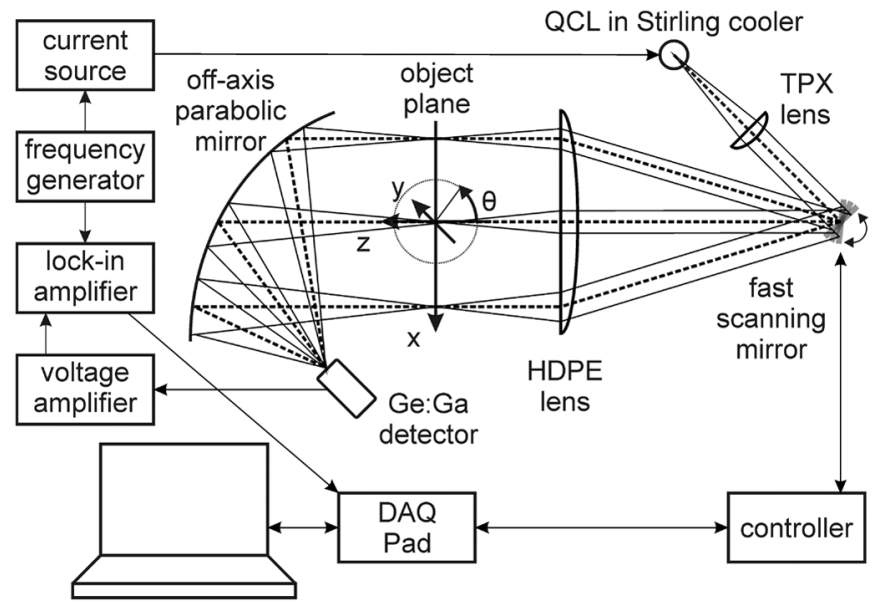

Fig. 1. Scheme of the experimental setup. The THz radiation emitted by the QCL is focused and scanned across the object. The transmitted radiation is detected with a Ge:Ga detector.

$\mathrm{kHz}$. The number of image pixels was chosen to correspond to the Nyquist sampling theorem of the resolution expected by the optical properties of the setup. According to this, the size of the elliptical image is 77 and 70 pixels in the horizontal and vertical direction, respectively. The value of one pixel is determined by averaging all samples of the scan trajectory within this pixel. The final image is normalized to a reference image without any object.

\section{Optical Properties of the IMAging System}

For the optimization of the spatial resolution and the size of the object plane, several parameters of the imaging system were considered. First of all, the fast scanning mirror forms the smallest aperture diameter in the system. This may cause influences on the beam profiles due to diffraction. In addition, the effective aperture is elliptical due to the $25^{\circ}$ angle of incidence. In the plane parallel to the optical table, the aperture size decreases with the cosine of the angle of incidence. In order to minimize this effect, the angle of incidence was chosen to be as small as possible, i.e. the reflected beam is not obstructed by the TPX lens.

The size of the object plane is limited by the focal length and the size of the HDPE lens. As mentioned above, the distance between the fast scanning mirror and the HDPE lens has to be equal to the focal length of the lens. Therefore, the size of the object plane is related to the focal length of the lens and the maximum deflection angle of the scanning mirror (cf. Fig. 1). In this case, the maximum deflection of $\pm 2.3^{\circ}$, which means a beam tilt of $\pm 4.6^{\circ}$, and the focal length of $250 \mathrm{~mm}$ results in an object plane size of $40 \mathrm{~mm}$ in the horizontal direction. The beam tilt in the vertical direction is only $\pm 4.2^{\circ}$ so that the object plane size is $36 \mathrm{~mm}$ in this direction. Although the deflection of the mirror is the same, the beam tilt is smaller in the vertical direction than in the horizontal direction due to the angle of incidence between the mirror and the beam.

In order to minimize diffraction effects at the edge of the lens, the size of the lens was chosen to be significantly larger than the object plane. Here, the minimum distance between the center of the outer beam and the edge of the lens is $17.6 \mathrm{~mm}$. In principle, it is possible to realize larger object planes. This requires a larger 


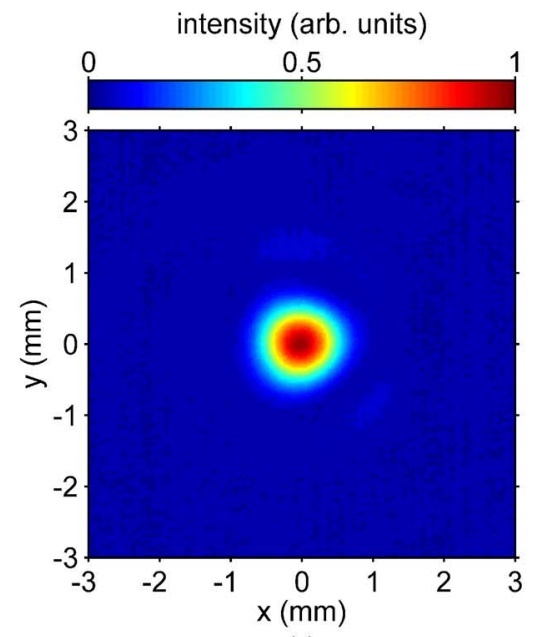

(a)

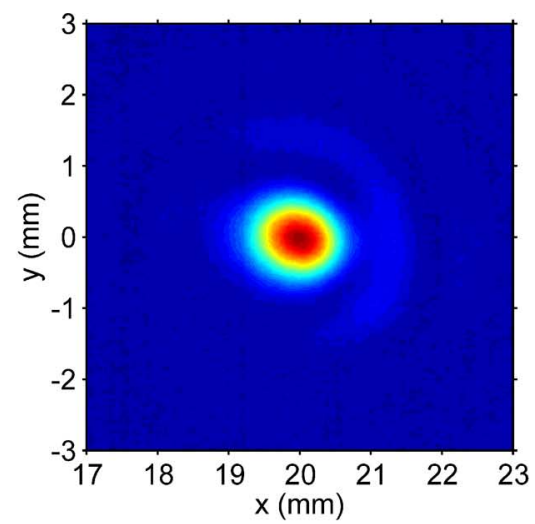

(b)

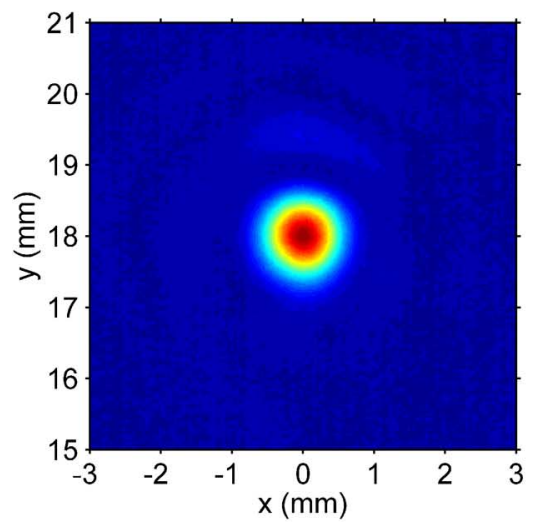

(c)

Fig. 2. Beam profiles in the object plane $(z=0)$ at (a) the center, (b) the right edge, and (c) the upper edge of the object plane measured with a microbolometer array camera. The change of the profiles within the object plane is moderate. The horizontal/vertical waist diameters are (a) $0.8 \mathrm{~mm} / 0.8 \mathrm{~mm}$, (b) $1.0 \mathrm{~mm} / 0.8 \mathrm{~mm}$, (c) and $0.8 \mathrm{~mm} / 0.8 \mathrm{~mm}$.

lens and a longer focal length which in turn results in a poorer spatial resolution.

The resolution of the imaging system is determined by the beam profiles shown in Fig. 2. The measurements of the beam profiles were performed with a commercial mid-infrared microbolometer camera (model Variocam from InfraTec). The camera consists of a microbolometer array with $640 \times 480$ pixels made of amorphous silicon. It is optimized for wavelengths

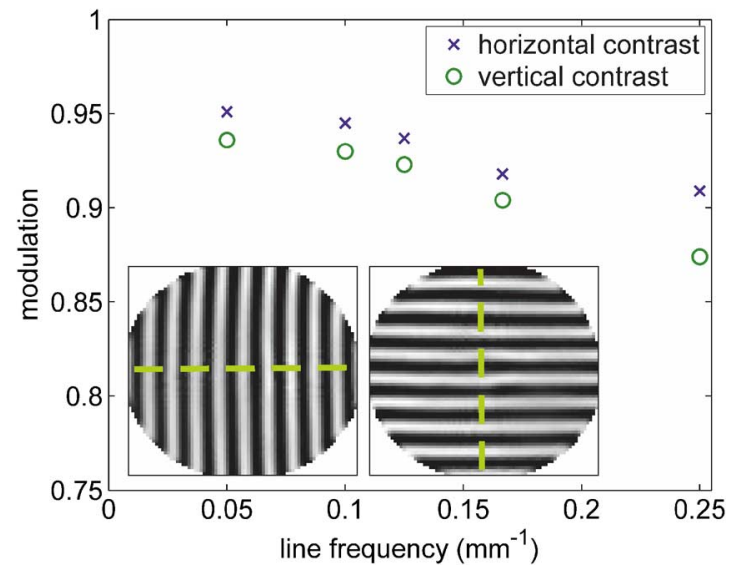

Fig. 3. Modulation transfer function and $\mathrm{THz}$ images (inset) of a metallic bar target with $2 \mathrm{~mm}$ wide bars and $2 \mathrm{~mm}$ spacing. The bars are resolved with contrasts of 0.91 and 0.87 in the horizontal and vertical direction, respectively.

of 8 to $14 \mu \mathrm{m}$. In order to improve its sensitivity at $\mathrm{THz}$ frequencies, the germanium objective lens in front of the camera is removed. The beam profiles shown in Fig. 2(a)-(c) were measured in the object plane on the optical axis, at the right edge, and at the upper edge of the object plane, respectively. There are only slight changes of the profile across the object plane, although the lens is used off axis, i.e. the beams pass through different areas of the lens. The beam waist diameters measured at different positions in the object plane are in the range of $0.8-1.0 \mathrm{~mm}$.

Another important figure of merit for evaluating the image quality is the contrast and its dependence on the spatial resolution, i.e., the modulation transfer function (MTF). In order to determine this, several metal bar targets with different numbers of bars per millimeter were imaged in the vertical and horizontal direction, and the contrast was determined. The contrast $C$ is defined by

$$
C=\frac{I_{\max }-I_{\min }}{I_{\max }+I_{\min }}
$$

where $I_{\max }$ and $I_{\min }$ denote the averaged values of the maximum and the minimum intensities, respectively, as determined from a line scan along the center of the image perpendicular to the bars (cf. dashed lines in the inset Fig. 3). The results are shown in Fig. 3 with the contrast being plotted as a function of the line frequency, which is defined as the number of bars per millimeter. In the inset, the THz images of a bar target with 0.25 bars per $\mathrm{mm}$ (bar width of $2 \mathrm{~mm}$ ) are shown. The bar target is well resolved with contrasts of 0.91 and 0.87 in the horizontal and vertical direction, respectively.

For 3-D CT imaging, the optical properties must be investigated over the whole imaged volume. The maximum volume of the 3-D image, which is calculated by the reconstruction algorithm, is determined by the 2-D object plane so that an ellipsoidal area with axes of 36 and $40 \mathrm{~mm}$ length in the vertical and horizontal direction, respectively, and with a $40 \mathrm{~mm}$ depth can be imaged. Fig. 4 shows the horizontal and vertical beam profiles along the $z$-direction through the center $(x=y=0)$, through the right edge ( $x=20 \mathrm{~mm}, y=0)$, and the upper edge $(x=0, y=18 \mathrm{~mm})$ of the object plane. Note that the beam paths are parallel with respect to each other, although they are 


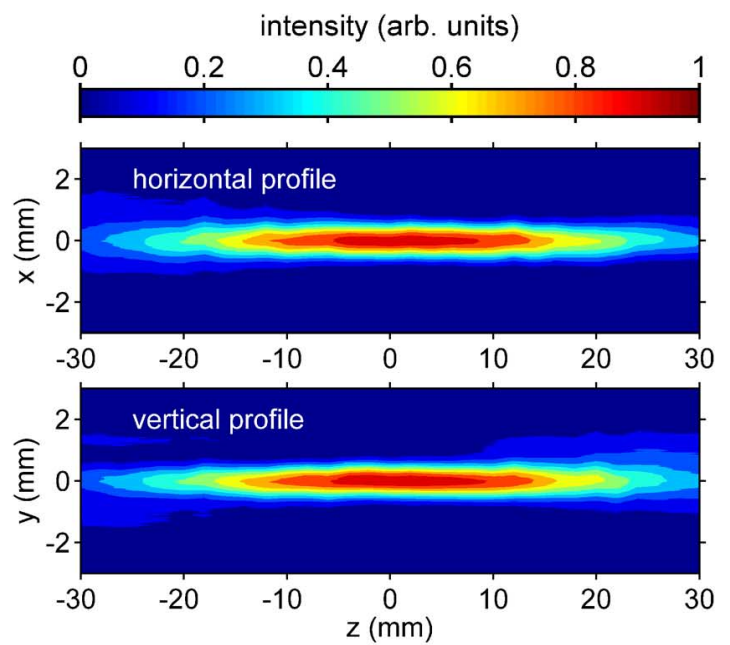

(a)
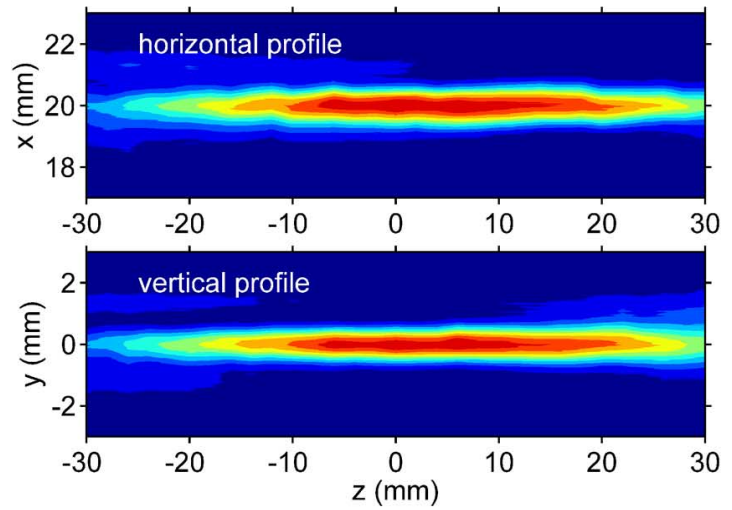

(b)
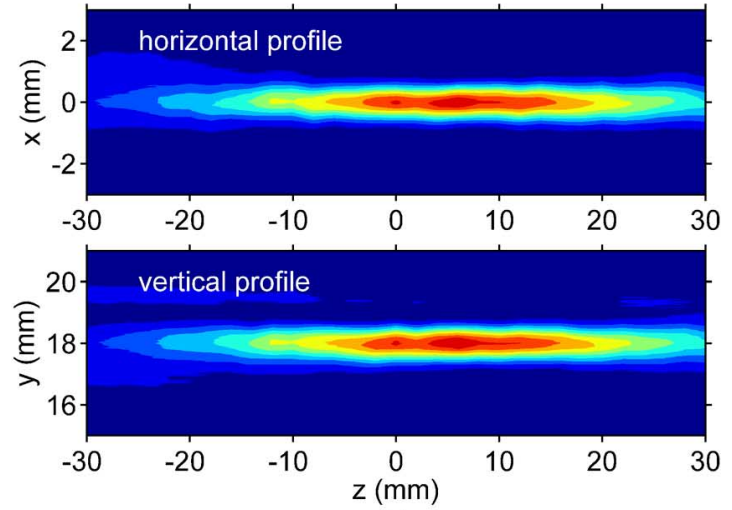

(c)

Fig. 4. Horizontal and vertical beam profiles along the $z$-direction through: (a) the center; (b) the right edge; and (c) the upper edge of the object plane.

deflected differently by the scanning mirror. This was achieved by the correct adjustment of the distance between the scanning mirror and the HDPE lens. A tilt of the beams may cause distortions in the reconstructed image, since the $\mathrm{CT}$ reconstruction algorithm assumes the beams to propagate parallel with respect to each other through the object.

The quality of the CT images crucially depends on the beam paths and the beam profiles within the imaged volume. The change of the profiles within the object volume is moderate. The waist diameter of all profiles varies from 0.8 to $1.2 \mathrm{~mm}$ within the imaged volume from $z=-20$ to $+20 \mathrm{~mm}$. The shape of the profiles within the measurement volume is well described by a Gaussian function. At both ends of this range, small side lobes appear. These can be seen for example in the vertical profiles in Fig. 4(b) and (c) from $z=-30$ to $-10 \mathrm{~mm}$. Since the measurement volume is an ellipsoid, these outer beams only pass a small area of the measurement volume around $z=0$. Therefore, the influence of the side lobes on the image quality is very small.

\section{2-D IMAGING}

The ultimate limitation of the imaging speed is determined by the mechanical movement of the scanning mirror. Therefore, the trajectory and the scanning speed of the mirror were thoroughly investigated and optimized. Scanning a rectangular object plane line by line leads to very high accelerations at the end of each line, resulting in a non-reproducible movement of the mirror and distorted images. Best results were achieved with an Archimedean spiral scan with a maximum deflection angle of $\pm 2.3^{\circ}$. In addition, the elliptical object plane resulting from this scan is better matched to the optical system because radiation from the corners of a rectangular scan is less efficiently coupled into the detector. The number of rotations which have to be performed by the mirror in order to obtain an image with a spatial resolution limited by the beam waist is determined by the size of the object plane. The beam waist diameter of about $1 \mathrm{~mm}$ and the diameter of the object plane of about $40 \mathrm{~mm}$ lead to 40 rotations of the mirror in order to obtain a Nyquist-sampled image. The measured data are transferred into an elliptical image with a total of 4550 pixels. It has a dimension of 77 by 70 pixels, according to the proportions of the object plane. Special care was taken on the algorithm, which transfers the data into the image, in order to avoid spiral artifacts in the image. The rotation frequency of the mirror is linearly increased from 20 $\mathrm{Hz}$ for large deflection angles corresponding to the outer border of the object plane to $60 \mathrm{~Hz}$ at the center. At even higher scanning frequencies, the trajectory of the mirror becomes unstable, and the object plane is not scanned homogeneously. This scanning scheme leads to an acquisition time of $1.1 \mathrm{~s}$ for one image. The average measurement time for one pixel is $240 \mu \mathrm{s}$. However, since the scanning speed increases toward the center of the image, the integration time of the lock-in amplifier was set to $10 \mu \mathrm{s}$. Using longer time constants led to distorted images, i.e. the delay between the scanning mirror and the response time of the lock-in amplifier was visible as spiral distortions in the image. With this data acquisition scheme, a signal-to-noise ratio of $28 \mathrm{~dB}$ has been obtained in the central part of the object plane, which decreases to $24 \mathrm{~dB}$ at the edges.

In Fig. 5(a), a THz image of a razor blade, which was covered by a sheet of paper, is shown. The brighter the image, the larger is the transmission. The shape of the blade is easily recognizable. Note that there is no fringe pattern in the image, which is often observed in images obtained when using a detector array [19], [22]. In Fig. 5(b), the intensity profile along the line in Fig. 5(a) is shown. One can clearly see the three transmission levels of the air, the paper, and the blade with values of approximately $1,0.45$, and 0 , respectively.

The photograph and the THz image of a 5 euros bill are displayed in Fig. 6(a) and (b), respectively. The THz image was 


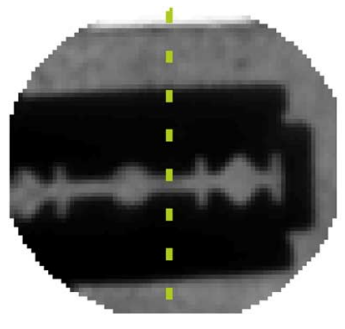

(a)

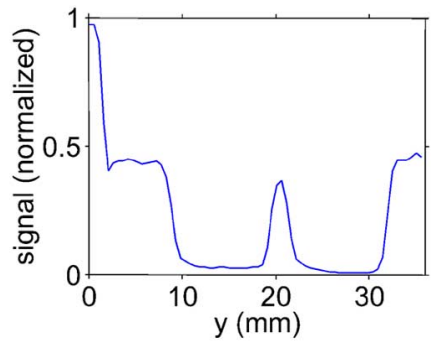

(b)
Fig. 5. (a) THz image of a razor blade covered by a sheet of paper and (b) intensity profile along the dashed line. The image was acquired by a spiral scan within $1.1 \mathrm{~s}$.

taken with a slightly modified setup. The HDPE lens was removed, whereas the TPX lens was positioned closer to the scanning mirror in order to focus the beam in the object plane. The image was acquired in the line scanning mode, where the mirror was repeatedly scanned along a horizontal line, while the bill was moved vertically with respect to the scanning line. The acquisition time of this image was 4 s. It consists of $132 \times$ 67 pixels, while the spatial resolution is similar to the one of the other setup. Several security-relevant features are visible in the $\mathrm{THz}$ image. Most of the features are separately shown in Fig. 6(c), for which the scaling of the gray values was individually adjusted for better clarity of the details. In area A, the watermark of the arc, which can be seen in the right part of the bill [Fig. 6(a)], is shown. The top and right columns of the arc are barely visible. Furthermore, the safety thread (B), the glossy stripe $(\mathrm{C})$, and the hologram (E) of the bill are recognizable as vertical stripes. The inhomogeneity in (C) is caused by alternate "euros" and "5" symbols which are embedded in the stripe. In the THz image, one can recognize that the symbols are different, but identification is not possible due to the limited spatial resolution. In (D), the lower part of the "5", which is printed on the bill, is visible. In the hologram stripe (E), an alternating transmission can be seen, which is caused by alternating holograms in the stripe.

\section{COMPUTED TOMOGRAPHY}

Based on the spirally scanning imaging setup, $\mathrm{THz}$ CT images were obtained from a series of projection images taken from different illumination angles using the rotation stage as described above. The object was placed on the stage and rotated by $180^{\circ}$ in steps of $3^{\circ}$. At each angular position, a 2-D image was obtained in the same manner as described in the previous section resulting in a total of 602 -D images with 4550 pixels each. At the outer edge of the imaged volume, one angular step corresponds to a translational displacement of $1.0 \mathrm{~mm}$. This is nearly equal to the waist diameter, i.e. the change of two subsequent images is barely resolvable. In fact, decreasing the angular step size and increasing the number of 2-D images did not improve the quality of the $\mathrm{CT}$ image. That was proven by a comparison of cross-sectional images, which were reconstructed from the same data set, but with different step widths. The images reconstructed with a step width of $1^{\circ}$ and $3^{\circ}$ are barely distinguishable. The acquisition time of one 3-D image is $87 \mathrm{~s}$. This is significantly shorter than that of other THz CT systems [14], [26]. The transmittance data were processed using filtered back

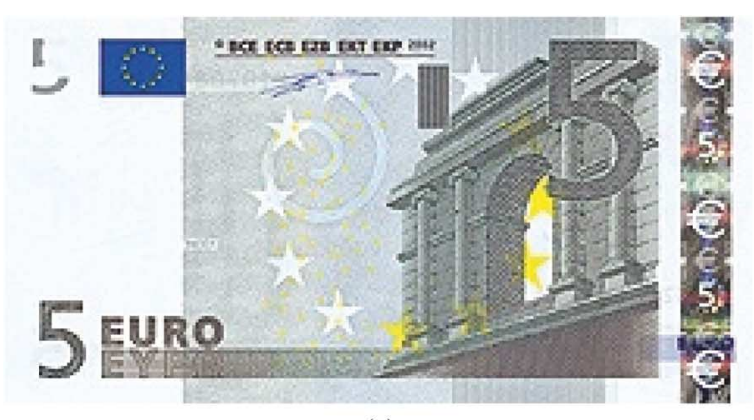

(a)

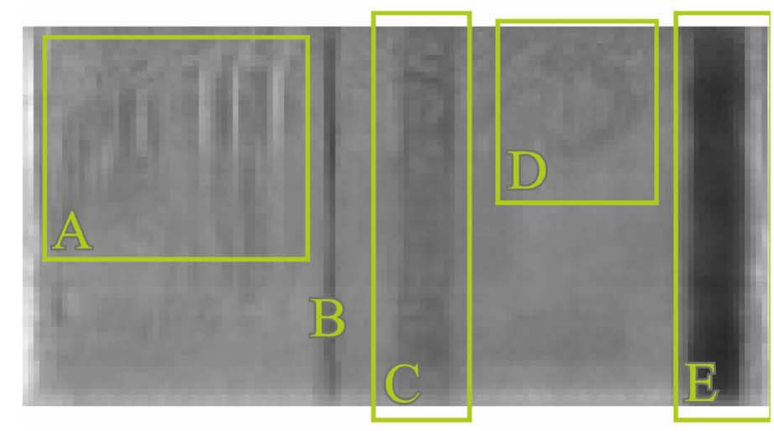

(b)

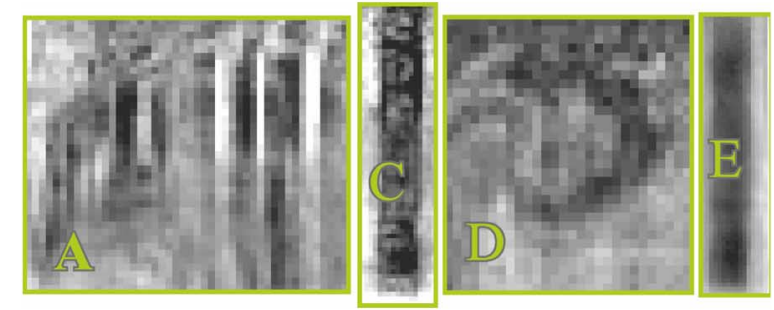

(c)

Fig. 6. (a) Photograph and (b) THz image of a 5 euros bill. Several securityrelevant features (A-E) can be seen. These are separately shown in (c). Note that in (c) the gray scale was adjusted separately for each part. The features are explained in the text.

projection, which is based on the reverse Radon transformation. It is important to keep the restrictions of this method in mind. First, the algorithm assumes a parallel projection, i.e. all transmitted beams from the QCL propagate parallel to each other. In Section III, it was shown that the optical setup fulfills this requirement. Second, the algorithm assumes that the loss of signal is purely due to absorption in the sample. At $\mathrm{THz}$ frequencies, this is generally not the case, because refractive index differences at interfaces, diffraction, and scattering affect the transmission through the sample [10], [27]. Therefore, meaningful CT images can only be obtained from samples where these influences are small. The influence of scattering effects on the reconstructed image is minimized by the setup, since the large parabolic off-axis mirror collects radiation, even when it is scattered by an angle up to $22^{\circ}$.

In Fig. 7, CT measurement results of a test object are presented. The test object consists of four rectangular frames made of HDPE with a height of $5 \mathrm{~mm}$ each. The frames are stacked on top of each other as shown in Fig. 7(a). The outer dimensions of all frames are $20 \times 25 \mathrm{~mm}^{2}$, while the inner dimensions decrease from top to bottom corresponding to an increasing wall thicknesses of 1, 2, 3, and $4 \mathrm{~mm}$. In Fig. 7(b), cross-sectional planes, 


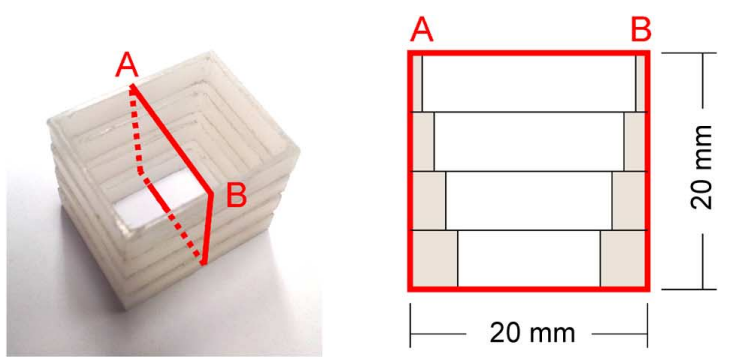

(a)

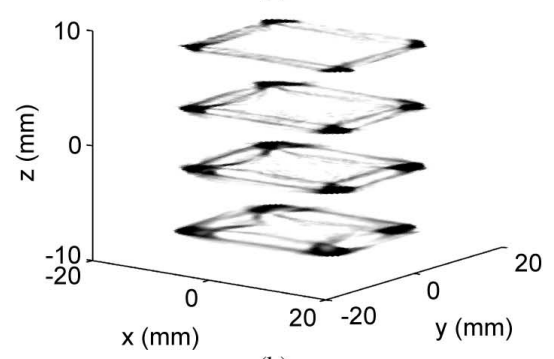

(b)
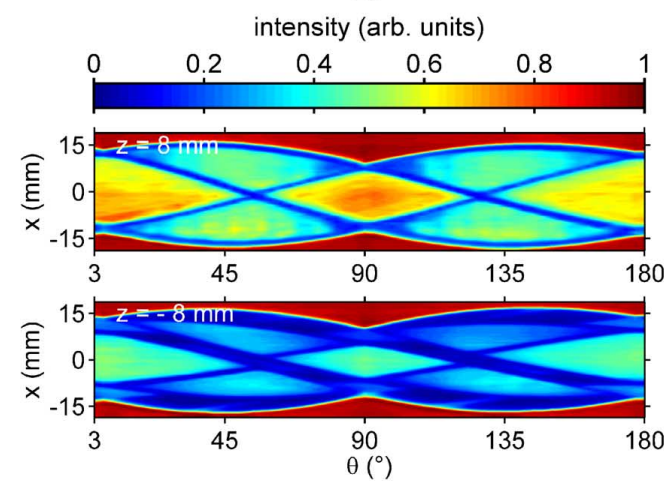

(c)

Fig. 7. (a) Schematic diagram of a test object, (b) CT measurement results of the object illustrated by cross-sectional planes, and (c) sinograms of the planes at $z=8 \mathrm{~mm}$ (top frame) and $z=-8 \mathrm{~mm}$ (bottom frame).

which are reconstructed from the measurements, are shown at different heights $(z=8,2.7,-2.7$, and $-8 \mathrm{~mm})$ of the test object corresponding to different frames, which are easily recognizable. The edges of the test object exhibit a reduced transmission due to increased reflection and scattering. In Fig. 7(c), the sinograms of the frames at $z=8 \mathrm{~mm}$ (wall thickness: $1 \mathrm{~mm}$ ) and $z=-8 \mathrm{~mm}$ (wall thickness: $4 \mathrm{~mm}$ ) are shown, which correspond to the intensity profiles in the $x$-direction as a function of the rotation angle $\theta$. At $\theta=0^{\circ}$ and $180^{\circ}$, the beam of the $\mathrm{QCL}$ is orthogonal to the long side of the frame, whereas at $\theta=90^{\circ}$ the beam is orthogonal to the short side of the frame. The upper sinogram represents a slice through the upper frame, where the wall thickness is $1 \mathrm{~mm}$. Four crossing lines are visible, which correspond to the edges of the frame. At the positions $\theta=0^{\circ}$, $90^{\circ}$, and $180^{\circ}$, the lines cross, since the edges of the object are perpendicular to the beam. From the minimum distance between the lines at $180^{\circ}$, the length of the long side of the frame is determined to be $25.5 \mathrm{~mm}$, while at $90^{\circ}$ the length of the short side is determined to be $20.4 \mathrm{~mm}$. This is in agreement with the dimensions of the test object within one pixel size accuracy of approximately $0.5 \mathrm{~mm}$. At $38.7^{\circ}$ and $141.3^{\circ}$, the beam is orthogonal to the diagonal axis of the frame resulting in the largest separation of the lines, which amounts to $32.2 \mathrm{~mm}$, again in agreement with the actual value of $32.0 \mathrm{~mm}$. At $51.3^{\circ}$ and $128.7^{\circ}$,

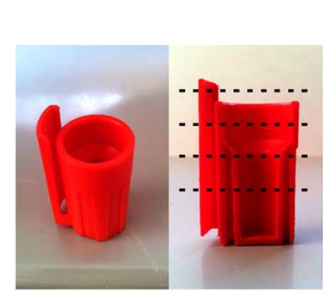

(a)

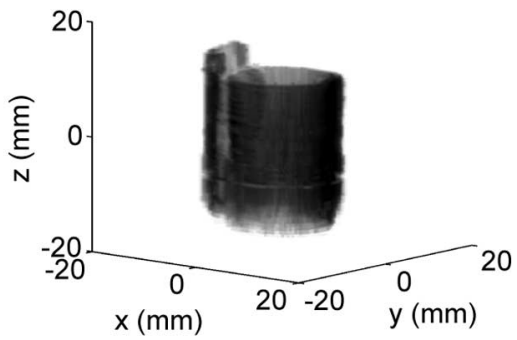

(b)

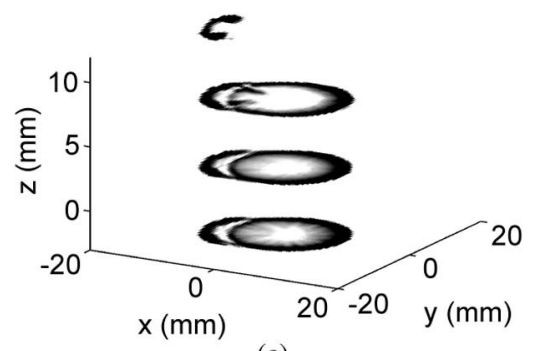

(c)

Fig. 8. (a) Photographs of a pen cap, (b) THz CT measurement result, and (c) four cross-sectional planes of the CT measurement. The dashed lines in (a) indicate the locations of the cross sections in (c).

two lines cross, since the diagonal axis is parallel with respect to the beam, i.e., two edges overlap in the projection. Between the lines, two different types of areas can be distinguished, one with a large transmission of approximately $70 \%$ and another one with a lower transmission of approximately $40 \%$. In the first area, the angle of incidence between the beam and the object interfaces is small. Therefore, the reflection losses are small, and the transmission value is in good agreement with the theoretical value of $70 \%$, assuming four reflection losses of $4 \%$ at each air-HDPE interface and a total absorption loss of $18 \%$ in the two $1 \mathrm{~mm}$ thick HDPE walls (assuming a refractive index of 1.53 and an absorption coefficient of $1 \mathrm{~cm}^{-1}[8]$ ). The second area corresponds to a larger angle of incidence. The measured transmission in this area is smaller for two reasons. First, the beam path through the walls is slightly longer, and, second, the reflection losses at the interfaces are higher. The lower sinogram can be interpreted in the same way. It represents the lowest frame with a wall thickness of $4 \mathrm{~mm}$. Thus, the lines in the sinogram are thicker. Again, the transmission of the object around the positions $\theta=0^{\circ}, 90^{\circ}$, and $180^{\circ}$ can be determined from the sinogram, which lies in the range between $40 \%$ and $50 \%$, whereas the theoretical value is $38 \%$.

In Fig. 8, results of a CT measurement of a more realistic object, namely a cap of a pen, are shown. Photographs of the cap and of a cross section through the cap, which was prepared after the measurement, are displayed in Fig. 8(a). The CT image is shown in Fig. 8(b), which consists of semi-transparent cross-sectional planes. One can clearly recognize the hollow volume in the center and the clip on the left side of the cap. In Fig. 8(c), four horizontal cross sections are displayed, the uppermost containing only the clip, while the three lower ones cut through the regions of the cap with different diameters of the hollow core [cf. dashed lines in Fig. 8(a)].

In Fig. 9(b), a CT image of a bag made from polyethylene filled with TPX pellets with about $4 \mathrm{~mm}$ diameter [cf. photograph in Fig. 9(a)] is presented, which consists of semi-transparent isosurfaces. The edges of the bag exhibit a reduced trans- 


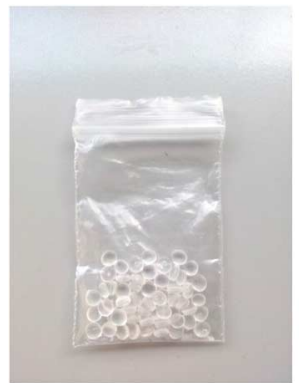

(a)

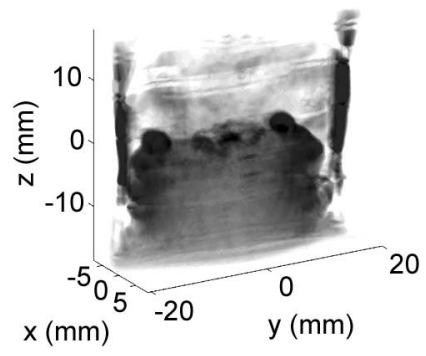

(b)
Fig. 9. (a) A bag filled with TPX pellets with approximately $4 \mathrm{~mm}$ diameter and (b) the corresponding $\mathrm{THz} \mathrm{CT}$ image.

mission due to the seam of the bag, which leads to increased reflection and scattering. Nevertheless, the CT image reveals the outer shape of the bag as well as the internal filling with the pellets. Near the edges, the transmission is higher, since the filling of the bag is not homogeneous, and near the edges are fewer pellets. Individual pellets can be distinguished on top of the filling as well as on the left side. In the center, the pellets are not distinguishable, since several pellets overlap. Note that the CT image contains more information than can be seen in the 2-D representation.

\section{SUMmary AND CONCLUSION}

A single-pixel detection system for fast $\mathrm{THz}$ imaging with a compact QCL source is demonstrated. With this system, it is possible to obtain transmission images with $0.5 \mathrm{~mm}$ spatial resolution within $1.1 \mathrm{~s}$. The SNR is as high as $28 \mathrm{~dB}$. Importantly, the image quality does not suffer from fringe artifacts. CT measurements with this system require as little as $87 \mathrm{~s}$ for an ellipsoidal imaging volume with approximately $40 \mathrm{~mm}$ long axes. The object dimensions derived from the $\mathrm{THz} \mathrm{CT}$ images are in agreement with the real dimensions of the object. The Ge:Ga detector, for example, might be replaced by a pyroelectric detector for further improvements. In addition, such a system offers an elegant implementation of sensitive spectroscopic capabilities since all the power from the QCL is always concentrated in one pixel. The scanning mirror can direct the $\mathrm{THz}$ beam to the area of interest, which was identified in the THz image, and this area might be spectroscopically analyzed with the same system just by adding a spectrometer in front of the detector [28].

\section{REFERENCES}

[1] C. S. Joseph, A. N. Yaroslavsky, V. A. Neel, T. M. Goyette, and R. H. Giles, "Continuous wave terahertz transmission imaging of nonmelanoma skin cancers.," Lasers Surg. Med., vol. 43, no. 6, pp. 457-462, Aug. 2011.

[2] C. Yu, S. Fan, Y. Sun, and E. Pickwell-Macpherson, "The potential of terahertz imaging for cancer diagnosis: A review of investigations to date," Quant. Imaging Med. Surg., vol. 2, no. 1, pp. 33-45, Mar. 2012.

[3] N. Karpowicz, H. Zhong, C. Zhang, K.-I. Li, J.-S. Hwang, J. Xu, and X.-C. Zhang, "Compact continuous-wave sub-terahertz system for inspection applications," Appl. Phys. Lett., vol. 86, no. 5, pp. 0541051-054105-2, Jan. 2005.

[4] K. B. Cooper, R. J. Dengler, N. Llombart, A. Talukder, A. V. Panangadan, C. S. Peay, I. Mehdi, and P. H. Siegel, "Fast, high-resolution terahertz radar imaging at 25 meters," Proc. SPIE, vol. 7671, pp. 76710Y1-76710Y-8, Apr. 2010.
[5] E. Heinz, T. May, D. Born, G. Zieger, G. Thorwirth, V. Zakosarenko, T. Krause, A. Krüger, M. Schulz, and H.-G. Meyer, "Toward high-sensitivity and high-resolution submillimeter-wave video imaging," Opt. Eng., vol. 50, no. 11, p. 113204, Nov. 2011.

[6] K. Kawase, Y. Ogawa, and Y. Watanabe, "Non-destructive terahertz imaging of illicit drugs using spectral fingerprints," Opt. Express, vol. 11, no. 20, pp. 2549-2554, Oct. 2003.

[7] Y. C. Shen, L. Gan, M. Stringer, A. Burnett, K. Tych, H. Shen, J. E. Cunningham, E. P. J. Parrott, J. A. Zeitler, L. F. Gladden, E. H. Linfield, and A. G. Davies, "Terahertz pulsed spectroscopic imaging using optimized binary masks," Appl. Phys. Lett., vol. 95, pp. 2311121-231112-3, Dec. 2009.

[8] E. Bründermann, H.-W. Hübers, and M. F. Kimmitt, Terahertz Techniques. Berlin, Heidelberg, Germany: Springer, 2012.

[9] W. L. Chan, J. Deibel, and D. M. Mittleman, "Imaging with terahertz radiation," Rep. Prog. Phys., vol. 70, no. 8, pp. 1325-1379, Jul. 2007.

[10] S. Wang and X.-C. Zhang, "Pulsed terahertz tomography," J. Phys. D: Appl. Phys., vol. 37, no. 4, pp. R1-R36, Jan. 2004.

[11] S. Busch, B. Scherger, M. Scheller, and M. Koch, "Optically controlled terahertz beam steering and imaging," Opt. Lett., vol. 37, no. 8, pp. 1391-1393, Apr. 2012.

[12] A. Dobroiu, M. Yamashita, Y. N. Ohshima, Y. Morita, C. Otani, and K. Kawase, "Terahertz imaging system based on backward-wave oscillator," Appl. Opt., vol. 43, no. 30, pp. 5637-5646, Oct. 2004.

[13] J. Darmo, V. Tamosiunas, G. Fasching, J. Kröll, K. Unterrainer, M. Beck, M. Giovannini, J. Faist, C. Kremser, and P. Debbage, "Imaging with a Terahertz quantum cascade laser," Opt. Express, vol. 12, no. 9, pp. 1879-1884, May 2004.

[14] K. L. Nguyen, M. L. Johns, L. Gladden, C. H. Worrall, P. Alexander, H. E. Beere, M. Pepper, D. A. Ritchie, J. Alton, S. Barbieri, and E. H. Linfield, "Three-dimensional imaging with a terahertz quantum cascade laser," Opt. express, vol. 14, no. 6, pp. 2123-2129, Mar. 2006.

[15] P. Dean, M. U. Shaukat, S. P. Khanna, S. Chakraborty, M. Lachab, A. Burnett, G. Davies, and E. H. Linfield, "Absorption-sensitive diffuse reflection imaging of concealed powders using a terahertz quantum cascade laser," Opt. Express, vol. 16, no. 9, pp. 5997-6007, Apr. 2008.

[16] S. Barbieri, J. Alton, C. Baker, T. Lo, H. E. Beere, and D. Ritchie, "Imaging with $\mathrm{THz}$ quantum cascade lasers using a Schottky diode mixer," Opt. Express, vol. 13, no. 17, pp. 6497-6503, Aug. 2005.

[17] U. S. de Cumis, J.-H. Xu, L. Masini, R. Degl'Innocenti, P. Pingue, F. Beltram, A. Tredicucci, M. S. Vitiello, P. A. Benedetti, H. E. Beere, and D. A. Ritchie, "Terahertz confocal microscopy with a quantum cascade laser source," Opt. Express, vol. 20, no. 20, pp. 21924-21931, 2012.

[18] P. Dean, Y. L. Lim, A. Valavanis, R. Kliese, M. Nikolić, S. P. Khanna, M. Lachab, D. Indjin, Z. Ikonić, P. Harrison, A. D. Rakić, E. H. Linfield, and G. Davies, "Terahertz imaging through self-mixing in a quantum cascade laser," Opt. Lett., vol. 36, no. 13, pp. 2587-2589, Jul. 2011.

[19] B. N. Behnken, G. Karunasiri, D. R. Chamberlin, P. R. Robrish, and J. Faist, "Real-time imaging using a $2.8 \mathrm{THz}$ quantum cascade laser and uncooled infrared microbolometer camera," Opt. Lett, vol. 33, no. 5, pp. 440-442, Mar. 2008.

[20] N. Oda, A. W. M. Lee, T. Ishi, I. Hosako, and Q. Hu, "Proposal for realtime Terahertz imaging system, with palm-size Terahertz camera and compact quantum cascade laser," Proc. SPIE, vol. 8363, pp. 83630A1-83630A-13, May 2012.

[21] A. W. M. Lee, B. S. Williams, S. Kumar, Q. Hu, and J. L. Reno, "Realtime imaging using a 4.3-THz quantum cascade laser and a $320 \times 240$ Microbolometer focal-plane array," IEEE Photon. Technol. Lett, vol. 18 , no. 13 , pp. $1415-1417$, Jul. 2006.

[22] N. Oda, T. Ishi, T. Morimoto, T. Sudou, H. Tabata, S. Kawabe, K. Fukuda, A. W. M. Lee, and Q. Hu, "Real-time transmission-type terahertz microscope with palm size terahertz camera and compact quantum cascade laser," Proc. SPIE, vol. 8496, pp. 84960Q-1-84960Q-11, Oct. 2012.

[23] H. Richter, A. D. Semenov, S. G. Pavlov, L. Mahler, A. Tredicucci, H. E. Beere, D. A. Ritchie, K. S. Ilin, M. Siegel, and H.-W. Hübers, "Terahertz heterodyne receiver with quantum cascade laser and hot electron bolometer mixer in a pulse tube cooler," Appl. Phys. Lett., vol. 93, no. 14, pp. 141108-1-141108-3, Oct. 2008.

[24] H. Richter, M. Greiner-Bär, S. G. Pavlov, A. D. Semenov, M. Wienold, L. Schrottke, M. Giehler, R. Hey, H. T. Grahn, and H.-W. Hübers, "A compact, continuous-wave terahertz source based on a quantum-cascade laser and a miniature cryocooler," Opt. Express, vol. 18, no. 10, pp. 10177-10187, May 2010. 
[25] S. Barbieri, J. Alton, H. E. Beere, J. Fowler, E. H. Linfield, and D. A. Ritchie, "2.9 THz quantum cascade lasers operating up to $70 \mathrm{~K}$ in continuous wave," Appl. Phys. Lett., vol. 85, no. 10, pp. 1674-1676, Sep. 2004.

[26] B. Ewers, A. Kupsch, A. Lange, B. R. Müller, A. Hoehl, R. Müller, and G. Ulm, "Terahertz spectral computed tomography," in 34th Int. Conf. on Infrared, Millim., and THz Waves, Sep. 2009.

[27] B. Recur, J. P. Guillet, I. Manek-Hönninger, J. C. Delagnes, W. Benharbone, P. Desbarats, J. P. Domenger, L. Canioni, and P. Mounaix, "Propagation beam consideration for 3D THz computed tomography," Opt. Express, vol. 20, no. 6, pp. 5817-5829, Mar. 2012.

[28] R. Eichholz, H. Richter, S. G. Pavlov, M. Wienold, L. Schrottke, R. Hey, H. T. Grahn, and H.-W. Hübers, "Multi-channel terahertz grating spectrometer with quantum-cascade laser and microbolometer array," Appl. Phys. Lett., vol. 99, no. 14, pp. 141112-1-141112-3, Oct. 2011.

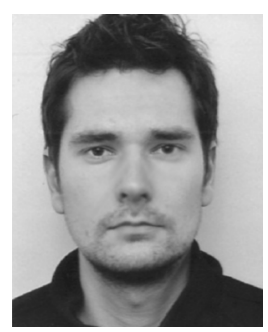

Nick Rothbart was born in Berlin, Germany, in 1985. He received the M.Sc degree in physics from Humboldt-Universität, Berlin, Germany, in 2011. Since 2011 he has been working towards the Ph.D. degree at the Department of Experimental Planetary Physics of Deutsches Zentrum für Luft- und Raumfahrt (DLR), Berlin, Germany. His current research involves the design and characterization of a $\mathrm{THz}$ imaging system.

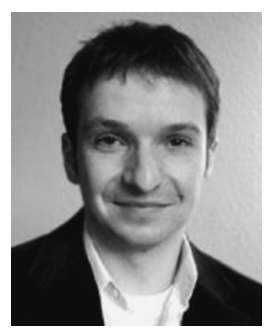

Heiko Richter received the Diploma degree in physics from the University of Karlsruhe, Karlsruhe, Germany, in 1999, and the Ph.D. degree in physics from the Technical University of Berlin, Berlin, Germany, in 2005. He is currently with the German Aerospace Center, Berlin, Germany, where he is involved in the field of terahertz and infrared sensors/optics. In 2007 he received the Lilienthal Award for the development of a $\mathrm{THz}$ security scanner.

Martin Wienold received his Diplom and doctoral degree in physics in 2007 and 2012, respectively, from the Humboldt-Universität zu Berlin, Germany, working on mid-infrared and THz quantum-cascade lasers. Since 2012, he is a postdoctoral research assistant at the Paul-Drude-Institut für Festkörper-elek- tronik in Berlin, Germany, working on the development of THz quantum-cascade lasers for spectroscopic applications.

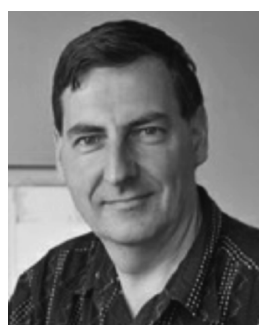

Lutz Schrottke received the Diplom and doctoral degrees in experimental physics in 1983 and 1988, respectively, from the Humboldt-Universität zu Berlin, Germany.

From 1985 to 1991 , he was with the Zentralinstitut für Elektronenphysik in Berlin, Germany, working on thin-film electroluminescent devices. In 1992, he joined the Paul-Drude-Institut für Festkörperelektronik in Berlin, Germany, as a scientific staff member. He was a Visiting Scholar at the Department of Physics of the University of Michigan, Ann Arbor, from 1995 to 1996. His research interests include $\mathrm{THz}$ quantum-cascade lasers as well as optical and transport properties of semiconductor heterostructures.

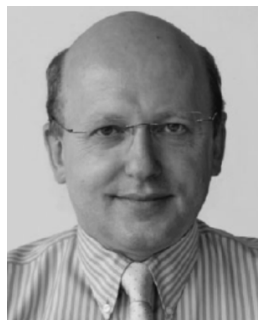

Holger T. Grahn received the Diplom degree in physics from the Universität Kiel, Germany, in 1983, and the Ph. D. degree in physics from Brown University, Providence, RI, USA, in 1987.

From 1988 to 1992, he was a Post-Doctoral research assistant at the Max-Planck-Institut für Festkörper-forschung in Stuttgart, Germany, working vertical transport in semiconductor superlattices. In 1992, he joined the Paul-Drude-Institut für Festkörper-elektronik in Berlin, Germany, as a department head, first for Analytics and later for Semiconductor Spectroscopy. In 2001, he was appointed Adjunct Professor in physics at the Technische Universität Berlin, Germany. His research interests include the optical and transport properties of semiconductor heterostructures and $\mathrm{THz}$ quantum-cascade lasers.

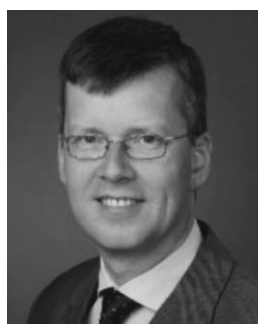

Heinz-Wilhelm Hübers received the diploma degree and Dr. rer. nat. degree in physics from the Universität Bonn, Germany, in 1991 and 1994, respectively.

From 1991 to 1994, he was with the Max-PlanckInstitut für Radioastronomie in Bonn, Germany. In 1994, he joined Deutsches Zentrum für Luft- und Raumfahrt (DLR) in Berlin, Germany, where he became head of department in 2001. Since 2009 he has been professor of experimental physics at the Technische Universität Berlin, Germany, and head of the department "Experimental Planetary Physics" at DLR. His research interests are in $\mathrm{THz}$ physics and spectroscopy, particularly in $\mathrm{THz}$ systems for astronomy, planetary research, and security.

Dr. Hübers received the Innovation Award on Synchrotron Radiation (2003) and the Lilienthal Award (2007). 